\title{
Interplay between Inflammaging, Frailty and Nutrition in Covid-19: Preventive and Adjuvant Treatment Perspectives
}

\author{
A. Padilha de Limal, , M. Macedo Rogero ${ }^{3}$, T. Araujo Viel ${ }^{4}$, H.M. Garay-Malpartida ${ }^{4}$, I. Aprahamian ${ }^{5}$, \\ S.M. Lima Ribeiro ${ }^{3,4}$
}

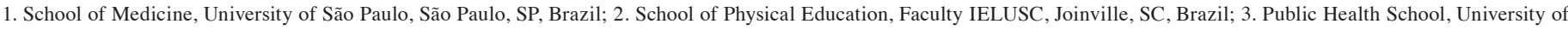

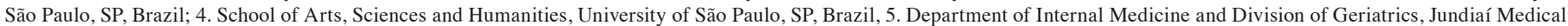
School, Jundiaí, SP, Brazil.

Corresponding Author: Sandra Maria Lima Ribeiro, University of São Paulo- Public Health School, Av Dr. Arnaldo 715, Sao Paulo- SP- Brazil, e-mail: smlribeiro@usp.br

\begin{abstract}
As humans age, their immune system undergoes modifications, including a low-grade inflammatory status called inflammaging. These changes are associated with a loss of physical and immune resilience, amplifying the risk of being malnourished and frail. Under the COVID-19 scenario, inflammaging increases the susceptibility to poor prognostics. We aimed to bring the current concepts of inflammaging and its relationship with frailty and COVID-19 prognostic; highlight the importance of evaluating the nutritional risk together with frailty aiming to monitor older adults in COVID-19 scenario; explore some compounds with potential to modulate inflammaging in perspective to manage the COVID-19 infection. Substances such as probiotics and senolytics can help reduce the high inflammatory status. Also, the periodic evaluation of nutrition risk and frailty will allow interventions, assuring the appropriate care.
\end{abstract}

Key words: COVID-19, inflammaging, nutritional risk, diet, probiotics, senolytics.

\section{Introduction}

oronavirus disease-19 (COVID-19), caused by Coronavirus 2 (SARS-CoV-2), has infected 219,893,295 persons worldwide by September 2nd, 2021, leading to 4,555,500 deaths (https://www.worldometers. info/coronavirus). The most remarkable difference between the SARS-CoV-2 and other SARS strains previously identified is its greater transmissibility and higher morbidity and mortality (1).

Despite the high transmissibility, most infected individuals remain asymptomatic (2) or present mild symptoms; a minor percentage present severe or critical manifestations $(2,3)$. The patient's global health status worsens in severe and critical manifestations. A dysfunctional systemic immune response occurs; this condition is frequently associated with a cytokine storm induced by SARS-CoV-2 (4). In addition, after hospital discharge, about $10 \%$ of the patients cannot return to the general health status they had before hospital admission or develop new clinical manifestations (5). These late manifestations have been called post-COVID-19 syndrome, and patients may present neurological, cardiovascular, and musculoskeletal disorders, burdening the functional status $(6,7)$. Several risk factors were implicated with the higher morbidity and mortality associated with COVID-19, such as higher age, obesity, diabetes, hypertension, coagulation dysfunctions, and inflammation disorders; organ damage involving the heart, liver, and kidneys are more associated with death (8).

The systemic inflammatory picture is a fundamental contributor to most adverse outcomes in COVID-19 (4). In this context, older adults, especially those with comorbidities and above 85 years old, generally present a higher peak of viral load and mortality than younger ones. Besides, the response to vaccination is usually reduced in older adults $(9,10)$. Aging and inflammation are intimately connected. Besides a higher burden of comorbidities, older adults present a typical picture of low-grade systemic inflammation (LGSI). This condition, also called inflammaging (11), seems to constitute an immune system dysregulation, possibly due to a loss of resilience to different stressors throughout life (11). Inflammaging has been considered an explanation to various geriatric syndromes, including frailty. Frailty, in turn, is conceived as a biological syndrome characterized by a decrease in the physiological reserve to respond to stressors due to the progressive failure of multiple organ systems necessary to maintain homeostasis (12). Notably, the brain, the endocrine and the immune systems, and the nutritional status are critical components involved in this process, culminating in disability and mortality (13).

Taking into account the simultaneous conditions of COVID19 infection, inflammaging, and frailty, we aimed to: (i) bring the current concepts of inflammaging and its relationship with frailty and COVID-19 prognostic; (ii) highlight the importance of evaluating the nutritional risk together with frailty aiming to follow older adults in COVID-19 scenario, and; (iii) explore some compounds with potential to modulate inflammaging in perspective to manage the COVID-19 infection.

\section{Inflammaging and frailty as risk factors to adverse outcomes}

During the life span, the human being must deal with different stressors such as environmental (i.e., radiation, air 
pollution), biologics (i.e., microorganisms), or psychological. The coexistence with these stressors demands adaptation, remodeling, or regeneration capacity. It should involve bodily responses such as antioxidants, apoptosis, phagocytosis, as well as inflammatory, neuroendocrine, and behavioral mechanisms (11).

Therefore, in the life course towards aging, damages accumulate, culminating in loss of resilience. This statement is coherent with the vicious frailty cycle proposed by Fried \& Watson (13), where the immune system assumes an important role $(14,15)$. Antigenic stimulus throughout life can lead to an imbalance in the immune system and consequent lowgrade systemic inflammation (inflammaging), which have been associated with many age-related conditions and diseases (11), including frailty.

Different models have been adopted to conceptualize frailty $(12,16)$. Despite their differences, all the models are associated with adverse outcomes such as disability, falls, hospitalization, and death (12). These concepts align with the modern geroscience theory of aging underpinned by mechanisms that stand out the LGSI and immune dysfunction (17). Therefore, frailty can be seen as a clinical measure of the degree of accelerated aging and inflammaging as a downstream pathway of this process.

\section{Contributors to inflammaging: cell senescence and molecular damage patterns}

Besides inflammaging, aging is featured by many other biological processes, for instance, cellular senescence. Cell senescence is characterized by a permanent growth-arrested stage; senescent cells are featured by resistance to apoptosis (18) and increased metabolic activity (19). Some hallmarks of senescent cells have been identified, including different factors of tissue damage, altered stem and progenitor cells, hemostasis, altered growth factors, and increased secretion of inflammatory cytokines and chemokines (i.e., IL-6, IL-8, monocyte chemoattractant protein-1, and plasminogen-activated inhibitor-1). Along with these hallmarks, the cells acquire the so-called senescence-associated secretory phenotype (SASP), typically with inflammatory features (20).

Another characteristic of the aging phenotype is the reduced capacity of cell machinery to recognize, metabolize or eliminate damage patterns derived from oxidative stress, inflammation, autophagy, and aged stem cells (21). These patterns can be apoptotic cells, antigenic molecules and organelles, metabolites, cell debris, and reactive species (22). These patterns, defined as damage-associated molecular patterns (DAMPs), are recognized by immune cells, resulting in activation of inflammasomes (18) and consequent secretion of cytokines (i.e., Il-1 $\alpha$ IL-1 $\beta$, IL-6, IL-8, and IL-18) $(23,24)$. Franceschi et al. (24) named these DAMPs as self-damage signals. Therefore, cell senescence and DAMPs generation are interrelated $(25,26)$.

\section{Contributors to inflammaging: the gut}

The gut microbiota has a crucial role in inflammaging. It can release inflammatory products, which Franceschi et al. (24) named quasi-self-damage signals. Many agingrelated factors can modify the gut microbiota, favoring the growth of pathogenic bacteria (i.e., Fusobacteria, Clostridia, Eubacteria) (27) and the reduction of some beneficial bacteria (i.e., Firmicutes, Bacteroides, and Bifidobacterium). These beneficial bacteria are featured by the ability to ferment polysaccharides and originate short-chain fatty acids (SCFA) $(28,29)$. The SCFAs, besides several metabolic functions, have fundamental immunological roles: (i) are the primary energy substrate to gut epithelial cells (29); (ii) regulate the secretion of cytokines and chemokines and are capable of inhibiting some inflammatory pathways (i.e., NF- $\varkappa \mathrm{B}$ and TNF- $\alpha$ expression) ; (iii) can improve the antigens presentation and differentiation of naïve CD4+ T cells into Tregs (30-33); (iv) help neutrophils chemotaxis and regulate the production of reactive oxygen species (ROS) by these cells $(34,35)$; and (v) in B cells, stimulate antibody production (36). Because of all these factors, bacterial unbalance and the reduced production of SCFA are associated with abnormal activation of the gut-associated lymphoid tissue (GALT), modifying the tolerance pattern of the immune cells $(37,38)$.

Changes in the gut environment harm its barrier function, allowing the passage of bacterial fragments (i.e., lipopolysaccharide-LPS) to the blood flow $(39,40)$. These fragments bind to specific pattern-recognizing receptors (PRRs), mainly the toll-like receptor-4 (TLR-4); these bindings trigger inflammatory signaling cascades that ultimately stimulate innate immune responses with a generation of proinflammatory cytokines, chemokines, eicosanoids, and ROS $(41,42)$. It is essential to highlight that the production of inflammatory cytokines constitutes a feedback cycle since these molecules stimulate the activity of TLRs (41). This inflammatory picture is named metabolic endotoxemia (43) and brings local and systemic consequences. For instance, it impairs endothelial cell function and contributes to atherosclerosis (44, $45)$; it leads to hepatic steatosis and insulin resistance (46); in the pancreas, it favors the $\beta$ cell dysfunction (47); in skeletal muscle, it increases the risk of sarcopenia $(45,48,49)$; and in the brain, it increases the neuroinflammation (50).

\section{Contributors to inflammaging: adipose tissue and dietary fat}

The aging-related increase and redistribution of body fat stand out in visceral white adipose tissue (44). The high-fat content in this tissue allows the infiltration of M1 macrophages and $\mathrm{T}$ lymphocytes. These immune cells, and the adipocytes themselves, release cytokines and chemokines (i.e., MCP-1, IL-1 $\beta$, IL- 6 , and TNF- $\alpha$ ), which settles an inflammatory profile $(51,52)$. In addition to these processes, the existing adipose tissue vasculature does not support the tissue's expansion, reducing the entry of oxygen and activating the hypoxiainducible factor-1 (HIF-1) that targets inflammatory genes. 
Systemically, the increase in body fat entails higher cellular exposition to saturated fatty acids (SFA), indirectly activating the TLRs signaling, possibly altering macrophage lipid metabolism (53). In addition, the excessive intake of lipids, especially SFA, can modify the gut microbiota contributing to metabolic endotoxemia. On the contrary, $\omega-3$ fatty acids seem to attenuate LPS and the SFA's effect in different tissues (54).

The contribution of the gut, adipose tissue, and diet to inflammaging raises the concept of metaflammation. Metaflammation is a metabolic disorder featured by cellular oxidative stress and increased plasma pro-inflammatory cytokine levels, principally triggered by nutrients and metabolic surplus; obesity is one of its main risk factors. The role of metabolic inflammation is well established in the pathogenesis of insulin resistance, mainly through stimulation of the proinflammatory cell signaling pathway, with activation of the transcription factors, namely nuclear factor-kappa $\mathrm{B}$ (NF-kB) and activator protein-1 (AP-1) mediated by toll-like receptor-4 (52-54). Therefore, inflammaging and metaflammation share similar molecular mechanisms (55).

\section{Immunosenescence and inflammaging}

Immunosenescence is a state of functional decline of the immune system, involves both the adaptive and innate immune arms, and is related to aged bone marrow and thymus involution $(55,56)$. Some features of immunosenescence will be briefly described. In the innate arm, the neutrophils show reduced chemotaxis, phagocytic activity, and intracellular killing of the pathogens. These cells also present defective ROS production $(57,58)$. The macrophages become hyperreactive due to inflammatory cytokines in blood flow. Dendritic cells are reduced (59), and these cells diminish phagocytosis and cytokines synthesis $(60,61)$. The natural killer cells (NK) have their number reduced, and the existing ones proliferate less (62), with reduced cytotoxicity and cytokine/chemokine synthesis. These changes result in a weakened response to pathogens.

There is a decline in lymphopoiesis and consequent reduced differentiation and proliferation of $\mathrm{T}$ - and $\mathrm{B}$ - cells concerning adaptative immunity. The B-cells, precursor, and their antibody-producing plasma cells decrease in number (63). Consequently, the "immunological space" is filled with memory cells with low receptor diversity; there is also a reduction in the T-cell diversity because the class-switching is reduced or impaired. Hence, there is a shrinking of the naive CD4+ T-cells, altering the CD4+/CD8+ ratio. These processes lead to difficulties in dealing with new antigens and weakened responses to vaccination (64).

An intriguing aspect of immunosenescence is the increased production of regulatory subtypes of myeloid and lymphoid cells. Typically, these cells inhibit the function of other immune cells induced by pathological and inflammatory conditions. Therefore, they are fundamental to the resolution of acute inflammation. However, in chronic inflammation and inflammaging, regulatory cells are induced in a compensatory immunosuppressor network involving diverse subgroups of regulatory cells. This regulatory phenotype leads some authors to define immunosenescence as different from the typical cell senescence, being a compensatory remodeling to inflammaging $(65,66)$.

\section{Covid-19 pathology and its relationship with frailty and inflammaging}

The SARS-CoV-2 virus enters the body via nasal cells, and its main targets are the alveolar epithelial type II cells. Initially, the viral spike " $S$ " protein binds the angiotensinconverting enzyme-2 (ACE-2) receptor. Afterward, the host's type 2 transmembrane serine protease (TMPRSS2) cleaves the ACE-2 and activates the " $\mathrm{S}$ " protein, promoting viral uptake and endocytosis. The virus releases its RNA and replicates using the host's machinery from its entry into other cells. From this point, the host generates new viruses, allowing the infection to progress.

Depending on the host's immune resilience, the virus reaches the lung, triggering a local immune response. The delayed-type I interferon increases the virus' sensing; the alveolar epithelial cells release monocyte chemoattractant, increasing, therefore, the monocytes and neutrophils into the lungs and promoting an exacerbated release of pro-inflammatory cytokines, chemokines, and growth factors. Systemic inflammation leads to vasodilation, contributing to the infiltration of monocytes and lymphocytes in the heart and lung. These responses decrease the interferon levels, raise neutrophil extracellular traps, and increase pyroptosis. The pyroptosis, in turn, increases the activity of NOD-like receptor protein-3 inflammasome (NLRP3), which releases several cytokines and chemokines, featuring the cytokine storm (i.e., IL-1 $\beta$, IL-2, IL-2R, IL-6, IL-7, IL-8 IL-10, IP10, MIP1A, and TNF- $\alpha)(2,67,68)$. The cytokine storm can cause a leak of nuclear or mitochondrial DNA (mtDNA), which is recognized by PRRs, especially in macrophages (69), increasing the inflammatory status. The occurrence of lung edema can fill the alveolar space, leading to the early-phase acute respiratory distress syndrome. This syndrome reduces the oxygen uptake, causing diffusing intravascular coagulation and thrombotic complications increasing the risk of sepsis and multiorgan failure and deaths.

Therefore, the SARS-CoV-2 infection demands a responsive and resilient immune system. In this scenario, it becomes clear that inflammaging and immunosenescence increase the vulnerability to poor prognostic $(70,71)$. The risk is increased in older adults with chronic diseases because they favor mechanisms by which the SARS-CoV-2 escapes the immune surveillance. Older adults deserve special attention at the COVID-19 pandemic. However, age and multimorbidity cannot be the only measures for severity or prognosis. The inflammaging increases the susceptibility to infection, augment the possibility of aggravating, and enhance the difficulty of recovering after the COVID-19 disease; thus, a permanent screening of indicators of physical resilience is crucial. In this perspective, frailty and nutrition must be considered.

During the COVID-19 pandemic, frailty, a clinical expression of vulnerability to immune dysfunction, is an 
essential measure for personalized care and resource allocation. The degree of frailty presents a dose-response relation to COVID-19 mortality (72-74). A recent meta-analysis observed that the Clinical Frailty Scale, a standard multidimensional screening instrument for frailty, was associated with a hazard ratio for mortality of 1.87 (75). Moreover, frailty points to a severity measure of COVID-19. The frailer patients have a higher chance of evolving towards severe form, a more extended hospital stay, and worse or incomplete recovery $(76,77)$. Frailty is also more prevalent among those patients with delirium due to COVID-19, and it may predict mortality (78). Delirium is an excellent example of a limited brain physiologic reserve as a presenting symptom of frailty, and their association seems bidirectional (79). Among the patients with acute confusion, between $16 \%$ and $37 \%$ had delirium as a primary symptom, or no typical COVID-19 symptom or sign was observed (80). Moreover, frail patients present immune dysfunction and may have a lower response to vaccination (81).

On the other hand, COVID-19 may predispose older patients to frailty. Although not fully understood, the upregulated interleukin- 6 due to the cytokine storm caused by SARS-CoV-2 activates STAT3 by tyrosine phosphorylation which triggers inflammatory feedback through the JAK/STAT signaling pathway $(82,83)$. Downstream ROS causes oxidative damage, amplifies a systemic pro-inflammatory state, culminating in muscle dysfunction and frailty. A high inflammatory condition reduces appetite and consequently decreases food intake, which aggravates muscle loss and frailty.

In summary, frailty and COVID-19 have a bidirectional relation in how each variable has feedback for the rise of the other. In this sense, inflammatory dysfunction over the LGSI (inflammaging) can mediate the co-occurrence of frailty and COVID-19.

\section{Nutritional and functional status}

The aging process raises the risk of malnutrition, which can rapidly increase and aggravate the management of severe respiratory infections. Respiratory burden means a hypermetabolic condition, which increases energy expenditure and, consequently, nutrition requirements. Achieving these requirements is challenging due to anorexia, anosmia, and ageusia. A study conducted in Wuhan, China, including 413 patients with COVID-19 (346 severe and 67 critically ill), evaluated their nutritional risk (NR). The critically ill patients showed higher NR scores, significantly associated with worsening COVID-19; therefore, the more severe the COVID19 status, the higher prevalence of NR or undernutrition (84). Also, comorbidities, such as diabetes-related hormonal dysfunction, can favor the NR. It is associated with high fatality rates in older adults infected by COVID-19 $(85,86)$.

A lower metabolic rate and higher NR are essential components of the vicious cycle of frailty. These include NR, diseases, reduced food intake, reduced physical activity, and reduced muscle mass. Cederholm (173) highlighted the existing overlapping between frailty, sarcopenia, and malnutrition; these three conditions have in common an inflammatory profile (acute or chronic) and nutrition deficiencies. The presence of diseases can aggravate these conditions, increasing the risk of disabilities and even death. Importantly, these conditions are reversible if appropriately identified and treated. Older adults, being more prone to develop severe cases of COVID-19, should be evaluated for these conditions. Low-cost and practical strategies must be thought out and implemented before, during, and at the recovery of COVID-19 disease.

Maltese et al. (2020), in a systematic review, highlighted some important aspects to be accounted for the management of older adults in the COVID-19 scenario: (i) monitoring older adults in home isolation; (ii) including frailty in the analysis of vaccination responses; (iii) using frailty, instead of age, as a predictive outcome to clinical decisions; (iv) monitoring frailty together with symptoms and outcomes in post-COVID; (v) using frailty evaluation to plan physical exercises, to prevent or counteract the muscle losses, and to investigate some metabolic parameters to help the dietary planning; (vi) discussing possibilities to counteract the immunosenescence. Going beyond and following Cederholm (173), we would reinforce the importance of simultaneously evaluating frailty and nutritional risk. Rapid screening tools can improve the assessment process. Also, assessing dietary intake and associated factors (for instance, loss of appetite) would help plan interventions.

\section{Nutrients and non-nutrients compounds}

In the perspective of attenuating some manifestations of inflammaging and frailty, it is essential to recognize some modifiable factors to interfere with immune modulator strategies. In the present review, we will explore, as modifiable factors, the gut (considering probiotics administration) and the cell senescence (considering senolytics substances administration).

\section{The gut as a target: administration of probiotics}

SARS-CoV-2 directly or indirectly affects the gut microbiota, especially in older adults, causing symptoms similar to intestinal inflammation (i.e., nausea, diarrhea, loss of appetite, and abdominal pain) $(6,87,88)$. Also, gut-derived toxins can affect the lungs, aggravating respiratory syndrome (89). There is evidence that changes in the intestinal microbiota regulate viral infection through several mechanisms (9092). For instance, it has been mentioned that possibly, the hypoxemia observed in the course of the disease can lead to a decrease in some genera of probiotic bacteria (Bifidobacterium, Lactobacillus, and Eubacterium) and an increase in other pathogenic bacteria (93). The over-administration of antibiotics in the COVID-19 treatment protocols is also a relevant factor affecting the intestinal microbiota. Gastrointestinal (GI) disturbances with changes in microbiota affect up to $50 \%$ of the COVID-19 patients and $17.6 \%$ of the severe cases (94, 95). Zuo et al. compared COVID-19 infected and non-infected patients' microbiota, finding increased opportunistic bacteria (Collinsella aerofaciens, Collinsella tanakaei, Streptococcus infantis, and Morganella morganii) in the infected ones (96). 
Besides, $\mathrm{Gu}$ et al. investigated bacterial groups modified with SARS-CoV-2 infection and identified a reduction in genders related to SCFA production (Faecalibacterium, Fusicatenibacter, and Eubacterium hallii) (95).

Li et al. formulated their hypothesis under the statement that patients with hypertension have an increased risk for COVID19 and a high percentage of GI symptoms (97). They studied spontaneously hypertensive rats, who typically presented higher expression of ACE-2 and TMPRSS2 in the gut epithelium, and depleted SCFA producers' bacteria. The results showed that butyrate downregulates some essential genes for SARS-CoV2 infection. At the same time, SCFA upregulated some antiviral pathways. The authors concluded that the increased COVID19 risk in hypertension is partly due to the gut's cumulative depletion of butyrate-producing bacteria.

Additionally, Pascoal et al. infected healthy colon epithelial cells with the SARS-CoV-2. They found that the treatment with SCFAs reduced the expression of genes related to the detection and replication of viral molecules (i.e., RIG1, TMPRSS2, and the IFN $\lambda$ receptor). However, they did not find any difference in the viral load with SCFA treatment.

Several studies have explored the possibility of using probiotics in SARS-CoV-2 infection. A report published in February 2020 suggested using probiotics added to the usual treatment (98) to control the severity of the disease. Baude et al. recommended that some species of Lactobacillus, Bifidobacterium, and Pediococcus are essential for reducing the viral load (99). Other studies performed in China showed that some patients with COVID-19 exhibited intestinal microbial dysbiosis characterized by reduced genders such as Bifidobacterium and Lactobacillus, suggesting supplementation with pre- or probiotics to return to eubiosis, decreasing the risk of infection (100).

As with other infectious respiratory diseases, the probiotic regulatory role in COVID-19 depends on the strain's specificity and the health condition of the host immune system. The SARS-CoV-2 induces an acute inflammatory bowel response via ACE2 and TMPRSS2, characterized by infiltration of the mucosa of macrophages, neutrophils, and T cells (101). Due to this, it has been hypothesized that probiotics may have a beneficial role in patients with gastrointestinal symptoms and those with mild to moderate systemic symptoms without respiratory impairment and help to prevent disease progression. The National Health Commission of China suggested using probiotics as a complementary treatment for gastrointestinal symptoms, such as diarrhea and reducing the risk of secondary infections due to microbial translocation in severe cases of COVID-19 (98). These suggestions represent a complementary tool to decrease the inflammation related to SARS-CoV-2 and favor the recovery of damages to the intestinal mucosa through the modulation of the intestinal microbiota.

From the information above, we can infer some mechanisms related to probiotics in COVID-19: (i) direct interaction with the virus; (ii) stimulation of the innate mucosal immune response; (iii) reduction of intestinal permeability; (iv) production of antiviral metabolites; and (v) promotion of the systemic acquired immune response through a regulatory and anti-inflammatory effect, especially during the cytokine storm
(102, 103).

On the other hand, some caution is necessary since the administration of microorganisms to patients with critical illnesses can lead to any injury, such as exacerbating inflammation. For this reason, in patients with severe disease and critically ill patients, the administration of probiotics should be evaluated, and essential safety issues should be considered (104). In contrast, the increase in innate defenses can probably help prevent COVID-19 in the initial stage of infection, especially in older adults. Therefore, a prophylactic administration of probiotics could be justified by their ability to maintain the integrity of the GALT and bacterial eubiosis, necessary to inhibit the entry of the virus into intestinal cells (89). Until vaccines are massively administered, improving the intestinal microbiota profile through personalized nutritional supplementation may be one of the prophylactic ways in which the impact of COVID-19 can be minimized in immunocompromised older adults. However, robust clinical trials are needed to elucidate these hot topics and identify the most valuable and safe environment for the administration of probiotics. Several registered trials that aim to investigate the efficiency of probiotics in treating COVID-19 patients are ongoing (101).

\section{Cell senescence as a target: Administration of polyphenols}

The overproduction of pro-inflammatory cytokines can lead us to compare this cellular condition to a SASP profile. This hypothetical profile raises the possibility of administering senolytic substances. Senolytics are compounds that induce apoptosis of senescent cells, reducing chronic inflammation and oxidative stress; therefore, they potentially reduce the risk of chronic diseases, malnutrition, and frailty (25). Some nutrients or non-nutrient molecules, as well as synthetic or natural products, are considered senolytics. Recently, some groups proposed using senolytic substances as possible therapeutics for COVID-19 since senescent phenotype (SASP) is strongly related to increased respiratory depression in COVID-19 patients $(105,106)$.

Polyphenols are phytochemicals present in fruits, vegetables, and plant-derived beverages. They represent diverse compounds separated into classes according to their chemical structures, including phenolic acids, flavonoids, stilbenes, curcuminoids, and lignans (107).

Among the polyphenols, curcumin, a natural hydrophobic polyphenol derived from the rhizome of Curcuma longa, modulates several molecular targets, including NF- $x \mathrm{~B}$, and the expression of the induced genes by this transcription factor (i.e., COX-2, iNOS, VCAM-1, ICAM-1, TNF- $\alpha$, IL-1, IL-6, IL-8, IL-12, and interferon- $\gamma$ ). Curcumin increases antioxidant defense mechanisms by upregulating transcription and expression levels of antioxidant enzymes and improving mitochondrial function (108-111). Studies in vitro showed that $5 \mathrm{uM}$ curcumin presented senolytic properties with a reduction in some hallmarks of senescence (i.e., p16, IL-6, IL-8, MMP3, and MMP13) (112). However, curcumin has lower 
bioavailability, which compromises its senolytic activity. A combination with piperine, alginates, or nanocapsules improves its stability and bioavailability (113).

Curcumin has been considered a perspective for treating COVID-19 based on its antiviral properties (114). For instance, in India, Pawar et al. (2021) conducted a double-blind, randomized controlled trial with symptomatic adult patients with COVID-19. They compared two groups, the first receiving a commercial probiotics-based compound (containing lactic acid Bacillus and Vitamin B), and the second receiving $525 \mathrm{mg}$ curcumin with $2.5 \mathrm{mg}$ piperine). Both treatments were given in similar capsules, twice a day for 14 days from the day of admission. The authors found many clinical improvements in patients in the curcumin group; however, there were no significant differences from the probiotics group (115). Also, Valizadeh et al. (2020) studied 40 COVID-19 patients divided into two groups, where the first (20 patients) received $160 \mathrm{mg}$ of Nano-curcumin in four $40 \mathrm{mg}$ capsules daily for 14 days, and the control group (20 patients) received a placebo. They investigated the mRNA expression and cytokine secretion levels of IL-1 $\beta$, IL-6, TNF- $\alpha$, and IL-18 (116). The nano-curcumin group presented improved results, especially in IL-1 $\beta$ and IL-6 mRNA expression and secretion.

Resveratrol (3,45-trihydroxy-stilbene) is a phytoalexin composed of two phenolic rings linked by a double bond. This compound exists in two isoforms: trans-resveratrol and cisresveratrol, with trans-resveratrol being the most stable form, which is found in grapes, berries, red wine, peanuts, among other fruits and nuts. Resveratrol attenuates the expression of proinflammatory cytokines in cells stimulated with LPS. It reduces the activation of the transcription factors $\mathrm{NF}-x \mathrm{~B}$ and AP-1. Additionally, resveratrol potentiated insulin on glucose uptake via AMPK in skeletal muscle cells (117-119). Timmers et al. (2011) verified that resveratrol supplementation $(150 \mathrm{mg} /$ day) activated AMPK, increased SIRT1 and PGC- $1 \alpha$ protein levels, and improved mitochondrial muscle respiration on the fatty acid-derived substrate in the muscle of healthy, obese men (120). Besides, resveratrol reduced systolic blood pressure and the HOMA index.

In conditions caused by virus infections other than COVID19 , resveratrol decreased symptoms of the common cold in children (121) and reduced TNF-alpha levels and diarrhea due to rotavirus in piglets (122). Once the potential of resveratrol to inhibit the Middle East Respiratory Syndrome Coronavirus (MERS-CoV) replication was described (123), the use of this compound as an adjunctive treatment of COVID-9 was suggested as a possible perspective. Pasquereau et al. found reduced viral titer by $80 \%$ with resveratrol in another member of the coronavirus family, the (HCoV)-229E (124). In another in vitro study, Yang et al. verified the effects of resveratrol on SARS-CoV-2 replication in Vero cell culture. Resveratrol was incubated with Vero cells before and or after their infection with SARS-CoV-2 (125). The inhibitory rate in cells treated with resveratrol after SARS-CoV-2 infection was excessive at $98 \%$, similar to the full-time treatment (pre and after treatment together). These two preliminary studies suggest that resveratrol can inhibit SARS-CoV-2 infection in the in vitro cell culture. However, to resveratrol's role in treating Covid-19, double- blinded clinical trials should be considered in futures studies.

\section{The importance of preventive strategies in aging}

As demonstrated above, The SARS-CoV-2 is a virus with high transmissibility. Most infected individuals remain asymptomatic or present mild symptoms. However, specific population groups, including older adults, are more vulnerable to developing severe or critical manifestations of COVID-19. In the present manuscript, we demonstrated that inflammaging and frailty are important contributors to this increased vulnerability. Therefore, in a pandemic environment, lifestyle-based preventive strategies should be highlighted.

In this context, the anti-inflammatory potential of diet has been extensively studied, especially from the Mediterranean dietary pattern (MDP). Traditionally, the MDP is high in vegetables, legumes, fruits, nuts, and cereals (mainly unrefined); moderate to high in fish and olive oil; moderate in fermented dairy products, especially cheese and yogurt; low in red meat and derivatives; moderate in alcohol, particularly in the form of wine, with meals. This food pattern is also high in monounsaturated/saturated fatty acids, fiber, and different phytochemicals (126). Furthermore, the MDP includes fresh, locally produced, and not modified or minimal modifications from their natural state (127). As discussed throughout this manuscript, all these components play essential roles in modulating systemic inflammation, oxidative stress, and apoptosis (128).

It is interesting to analyze that the most recent studies on MDP have shown a view beyond simple food intake. New proposals have included environmental aspects and physical activity as an essential preventive and healthy lifestyle. It is known that decreased physical activity has a detrimental role in muscle quality, and inappropriate nutritional habits favor the acceleration of immunosenescence and inflammaging (129).

Physical activity can alter the systemic inflammation of older adults regardless of their level of intensity, reducing blood fibrinogen and C-reactive protein levels. It should be noted that all significant influences on fibrinogen and $\mathrm{C}$-reactive protein by displacement of different physical activity behaviors remained after adjustment for metabolic risk status among participants.

Physical inactivity has shown a strong association with an increased risk for severe COVID-19 outcomes. Sallis et al. carried out a cohort study with 48,440 adults showing that patients with COVID-19 who were consistently inactive had a greater chance of hospitalization, admission to the intensive care unit, and death than those who met the physical activity guidelines (130). A recently published international guideline on physical activity and exercise for older adults aims to facilitate the prescription of exercise by non-specialists according to several diseases or geriatric syndromes (131).

In Figure 1, we constructed a framework putting together inflammaging with associated factors and outcomes, together with the possibilities of preventing or assisting the treatment with nutrition. 
Figure 1. The interplay between malnutrition and chronic inflammation leads to a state of physiological vulnerability typical of unhealthy aging. An older adult in this condition has a greater susceptibility of COVID -19 aggravation after contact with Sars-Cov-2. Strategies such as diet and physical activity may prevent physiological vulnerability and are postulated to fight off COVID-19 aggravation. Also, those strategies can be explored to promote healthy aging and improve the management of diseases, increasing the probability of recovery with less severe sequelae

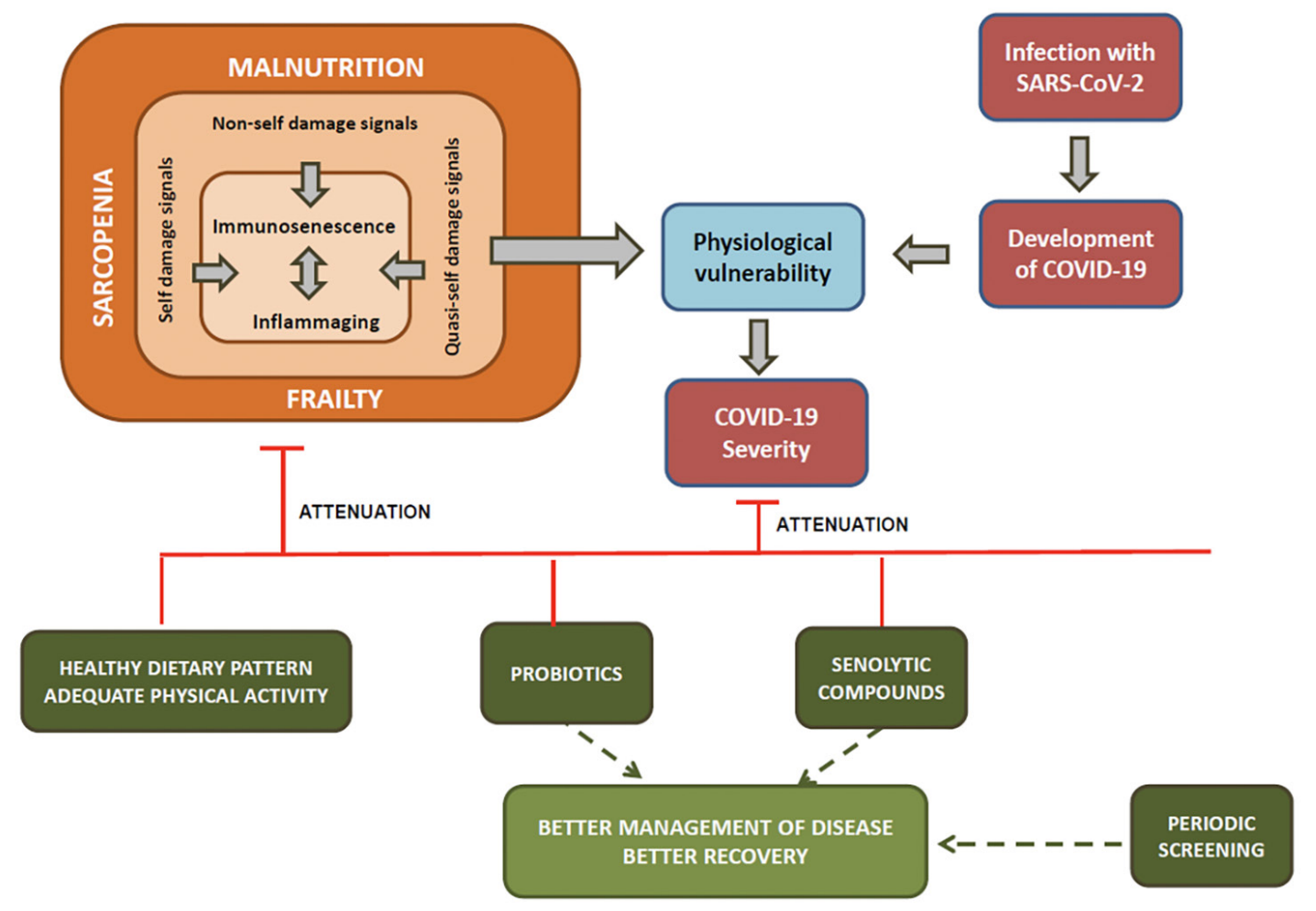

\section{Conclusion}

The inflammaging can trigger conditions such as malnutrition and frailty and, consequently, increase the vulnerability to COVID-19 aggravation. It is mandatory to monitor older adults concerning nutrition, functional status, and frailty. These evaluations will allow interventions, assuring the appropriate care. We highlighted the possibility of interventions with substances directed to control the inflammatory status via the gut or cell senescence.

Funding statement: This research did not receive any specific grant from funding agencies in the public, commercial, or not-for-profit sectors.

\section{Conflict of Interest: The authors declare no conflicts of interest.}

Authors' contribution: SMLR planned the manuscript; APL, MMR, TAV HMGM and SMLR searched the literature, wrote the manuscript, and revised the final version; IA revised the final version and contributed with a clinical practice point of view.

\section{References}

1. Kumar A, Singh R, Kaur J, Pandey S, Sharma V, Thakur L et al. Wuhan to World: The COVID-19 Pandemic. Front Cell Infect Microbiol. 2021;11:596201. doi: 10.3389/ fcimb.2021.596201.

2. Chen Y, Klein SL, Garibaldi BT, Li H, Wu C, Osevala NM et al. Aging in COVID19: Vulnerability, immunity and intervention. Ageing Res Rev. 2021;65:101205. doi: 10.1016/j.arr.2020.101205.

3. Silvin A, Chapuis N, Dunsmore G, Goubet AG, Dubuisson A, Derosa L et al. Elevated Calprotectin and Abnormal Myeloid Cell Subsets Discriminate Severe from Mild COVID-19. Cell. 2020;182:1401-18.e18. doi: 10.1016/j.cell.2020.08.002.

4. García LF. Immune Response, Inflammation, and the Clinical Spectrum of COVID-19. Front Immunol. 2020;11:1441. doi: 10.3389/fimmu.2020.01441.
5. Carfî A, Bernabei R, Landi F, Group GAC-P-ACS. Persistent Symptoms in Patients After Acute COVID-19. JAMA. 2020;324:603-5. doi: 10.1001/jama.2020.12603.*

6. Huang C, Wang Y, Li X, Ren L, Zhao J, Hu Y et al. Clinical features of patients infected with 2019 novel coronavirus in Wuhan, China. Lancet. 2020;395:497-506. doi: 10.1016/S0140-6736(20)30183-5.

7. Mao L, Jin H, Wang M, Hu Y, Chen S, He Q et al. Neurologic Manifestations of Hospitalized Patients With Coronavirus Disease 2019 in Wuhan, China. JAMA Neurol. 2020;77:683-90. doi: 10.1001/jamaneurol.2020.1127.

8. Wolff D, Nee S, Hickey NS, Marschollek M. Risk factors for Covid-19 severity and fatality: a structured literature review. Infection. 2021;49:15-28. doi: 10.1007/s15010020-01509-1.

9. Wu Z, McGoogan JM. Characteristics of and Important Lessons From the Coronavirus Disease 2019 (COVID-19) Outbreak in China: Summary of a Report of 72314 Cases From the Chinese Center for Disease Control and Prevention. JAMA. 2020. doi: 10.1001/jama.2020.2648.

10. Vellas C, Delobel P, de Souto Barreto P, Izopet J. COVID-19, Virology and Geroscience: A Perspective. J Nutr Health Aging. 2020;24:685-91. doi: 10.1007/ s12603-020-1416-2.

11. Franceschi C, Bonafè M, Valensin S, Olivieri F, De Luca M, Ottaviani E et al. Inflamm-aging. An evolutionary perspective on immunosenescence. Ann N Y Acad Sci. 2000;908:244-54. doi: 10.1111/j.1749-6632.2000.tb06651.x.

12. Hoogendijk EO, Afilalo J, Ensrud KE, Kowal P, Onder G, Fried LP. Frailty: implications for clinical practice and public health. Lancet. 2019;394:1365-75. doi: 10.1016/S0140-6736(19)31786-6.

13. Fried LP, Tangen CM, Walston J, Newman AB, Hirsch C, Gottdiener J et al. Frailty in older adults: evidence for a phenotype. J Gerontol A Biol Sci Med Sci. 2001;56:M14656. doi: 10.1093/gerona/56.3.m146.

14. Sansoni P, Vescovini R, Fagnoni F, Biasini C, Zanni F, Zanlari L et al. The immune system in extreme longevity. Exp Gerontol. 2008;43:61-5. doi: 10.1016/j. exger.2007.06.008

15. Hazeldine J, Lord JM. Innate immunesenescence: underlying mechanisms and clinical relevance. Biogerontology. 2015;16:187-201. doi: 10.1007/s10522-014-9514-3.

16. Dent E, Morley JE, Cruz-Jentoft AJ, Woodhouse L, Rodríguez-Mañas L, Fried LP et al. Physical Frailty: ICFSR International Clinical Practice Guidelines for Identification and Management. J Nutr Health Aging. 2019;23:771-87. doi: 10.1007/s12603-0191273-z. 
17. Kennedy BK, Berger SL, Brunet A, Campisi J, Cuervo AM, Epel ES et al. Geroscience: linking aging to chronic disease. Cell. 2014;159:709-13. doi: 10.1016/j. cell.2014.10.039.

18. Salminen A, Kaarniranta K, Kauppinen A. Inflammaging: disturbed interplay between autophagy and inflammasomes. Aging (Albany NY). 2012;4:166-75. doi: 10.18632/ aging.100444.

19. Kirkland JL, Tchkonia T. Cellular Senescence: A Translational Perspective. EBioMedicine. 2017;21:21-8. doi: 10.1016/j.ebiom.2017.04.013.

20. Tchkonia T, Zhu Y, van Deursen J, Campisi J, Kirkland JL. Cellular senescence and the senescent secretory phenotype: therapeutic opportunities. J Clin Invest. 2013;123:96672. doi: 10.1172/JCI64098.

21. López-Otín C, Blasco MA, Partridge L, Serrano M, Kroemer G. The hallmarks of aging. Cell. 2013;153:1194-217. doi: 10.1016/j.cell.2013.05.039.

22. Fulop T, Larbi A, Dupuis G, Le Page A, Frost EH, Cohen AA et al. Immunosenescence and Inflamm-Aging As Two Sides of the Same Coin: Friends or Foes? Front Immunol 2017;8:1960. doi: 10.3389/fimmu.2017.01960

23. Xia S, Zhang X, Zheng S, Khanabdali R, Kalionis B, Wu J et al. An Update on Inflamm-Aging: Mechanisms, Prevention, and Treatment. J Immunol Res. 2016;2016:8426874. doi: 10.1155/2016/8426874.

24. Franceschi C, Garagnani P, Parini P, Giuliani C, Santoro A. Inflammaging: a new immune-metabolic viewpoint for age-related diseases. Nat Rev Endocrinol. 2018;14:576-90. doi: 10.1038/s41574-018-0059-4.

25. Kirkland JL, Tchkonia T, Zhu Y, Niedernhofer LJ, Robbins PD. The Clinical Potential of Senolytic Drugs. J Am Geriatr Soc. 2017;65:2297-301 . doi: 10.1111/jgs.14969.

26. Lavasani M, Robinson AR, Lu A, Song M, Feduska JM, Ahani B et al. Muscle-derived stem/progenitor cell dysfunction limits healthspan and lifespan in a murine progeria model. Nat Commun. 2012;3:608. doi: 10.1038/ncomms 1611.

27. Woodmansey EJ. Intestinal bacteria and ageing. J Appl Microbiol. 2007;102:1178-86 doi: 10.1111/j.1365-2672.2007.03400.x.

28. Flint HJ, Duncan SH, Scott KP, Louis P. Links between diet, gut microbiota composition and gut metabolism. Proc Nutr Soc. 2015;74:13-22. doi: 10.1017/ S0029665114001463.

29. Levy M, Blacher E, Elinav E. Microbiome, metabolites and host immunity. Curr Opin Microbiol. 2017;35:8-15. doi: 10.1016/j.mib.2016.10.003

30. Park J, Kim M, Kang SG, Jannasch AH, Cooper B, Patterson J et al. Short-chain fatty acids induce both effector and regulatory $\mathrm{T}$ cells by suppression of histone deacetylases and regulation of the mTOR-S6K pathway. Mucosal Immunol. 2015;8:80-93. doi: 10.1038/mi.2014.44.

31. Smith PM, Howitt MR, Panikov N, Michaud M, Gallini CA, Bohlooly-Y M et al. The microbial metabolites, short-chain fatty acids, regulate colonic Treg cell homeostasis. Science. 2013;341:569-73. doi: 10.1126/science.1241165.

32. Wang D, Hu B, Hu C, Zhu F, Liu X, Zhang J et al. Clinical Characteristics of 138 Hospitalized Patients With 2019 Novel Coronavirus-Infected Pneumonia in Wuhan, China. JAMA. 2020;323:1061-9. doi: 10.1001/jama.2020.1585.

33. Berndt BE, Zhang M, Owyang SY, Cole TS, Wang TW, Luther J et al. Butyrate increases IL-23 production by stimulated dendritic cells. Am J Physiol Gastrointest Liver Physiol. 2012;303:G1384-92. doi: 10.1152/ajpgi.00540.2011.

34. Segal AW. How neutrophils kill microbes. Annu Rev Immunol. 2005;23:197-223. doi: 10.1146/annurev.immunol.23.021704.115653.

35. Vinolo MA, Rodrigues HG, Hatanaka E, Sato FT, Sampaio SC, Curi R. Suppressive effect of short-chain fatty acids on production of proinflammatory mediators by neutrophils. J Nutr Biochem. 2011;22:849-55. doi: 10.1016/j.jnutbio.2010.07.009.

36. Kim MK, Jeon JH, Kim SW, Moon JS, Cho NH, Han E et al. The Clinica Characteristics and Outcomes of Patients with Moderate-to-Severe Coronavirus Disease 2019 Infection and Diabetes in Daegu, South Korea. Diabetes \& Metabolism Journal. 2020;44:602-+. doi: 10.4093/dmj.2020.0146.

37. Amsterdam D, Ostrov BE. The Impact of the Microbiome on Immunosenescence. Immunol Invest. 2018;47:801-11. doi: 10.1080/08820139.2018.1537570.

38. André P, Laugerette F, Féart C. Metabolic Endotoxemia: A Potential Underlying Mechanism of the Relationship between Dietary Fat Intake and Risk for Cognitive Impairments in Humans? Nutrients. 2019;11. doi: 10.3390/nu11081887.

39. Cani PD, Bibiloni R, Knauf C, Waget A, Neyrinck AM, Delzenne NM et al. Changes in gut microbiota control metabolic endotoxemia-induced inflammation in high-fat diet-induced obesity and diabetes in mice. Diabetes. 2008;57:1470-81. doi: 10.2337/ $\mathrm{db} 07-1403$

40. Kamada N, Chen GY, Inohara N, Núñez G. Control of pathogens and pathobionts by the gut microbiota. Nat Immunol. 2013;14:685-90. doi: 10.1038/ni.2608.

41. Kim JJ, Sears DD. TLR4 and Insulin Resistance. Gastroenterol Res Pract. 2010;2010. doi: $10.1155 / 2010 / 212563$

42. Park BS, Lee JO. Recognition of lipopolysaccharide pattern by TLR4 complexes. Exp Mol Med. 2013;45:e66. doi: 10.1038/emm.2013.97.

43. Cani PD, Amar J, Iglesias MA, Poggi M, Knauf C, Bastelica D et al. Metabolic endotoxemia initiates obesity and insulin resistance. Diabetes. 2007;56:1761-72. doi: $10.2337 / \mathrm{db} 06-1491$

44. Zorena K, Jachimowicz-Duda O, Ślęzak D, Robakowska M, Mrugacz M. Adipokines and Obesity. Potential Link to Metabolic Disorders and Chronic Complications. Int J Mol Sci. 2020;21. doi: 10.3390/ijms21103570.
45. Livshits G, Kalinkovich A. Inflammaging as a common ground for the development and maintenance of sarcopenia, obesity, cardiomyopathy and dysbiosis. Ageing Res Rev. 2019;56:100980. doi: 10.1016/j.arr.2019.100980.

46. Baffy G. Kupffer cells in non-alcoholic fatty liver disease: the emerging view. J Hepatol. 2009;51:212-23. doi: 10.1016/j.jhep.2009.03.008.

47. Eguchi K, Nagai R. Islet inflammation in type 2 diabetes and physiology. J Clin Invest. 2017;127:14-23. doi: 10.1172/JCI88877.

48. Abbatecola AM, Paolisso G. Is there a relationship between insulin resistance and frailty syndrome? Curr Pharm Des. 2008;14:405-10. doi 10.2174/138161208783497750.

49. Y B. Physiopathological mechanism of sarcopenia. J Nutr Health Aging; 2009; 13: 717-23.

50. Di Benedetto S, Müller L, Wenger E, Düzel S, Pawelec G. Contribution of neuroinflammation and immunity to brain aging and the mitigating effects of physical and cognitive interventions. Neurosci Biobehav Rev. 2017;75:114-28. doi: 10.1016/j. neubiorev.2017.01.044

51. Lee YS, Wollam J, Olefsky JM. An Integrated View of Immunometabolism. Cell 2018; 172:22-40. doi: 10.1016/j.cell.2017.12.025

52. Hotamisligil GS, Erbay E. Nutrient sensing and inflammation in metabolic diseases. Nat Rev Immunol. 2008;8:923-34. doi: 10.1038/nri2449.

53. Nguyen MT, Favelyukis S, Nguyen AK, Reichart D, Scott PA, Jenn A et al. A subpopulation of macrophages infiltrates hypertrophic adipose tissue and is activated by free fatty acids via Toll-like receptors 2 and 4 and JNK-dependent pathways. J Biol Chem. 2007;282:35279-92. doi: 10.1074/jbc.M706762200.

54. Lee JY, Sohn KH, Rhee SH, Hwang D. Saturated fatty acids, but not unsaturated fatty acids, induce the expression of cyclooxygenase- 2 mediated through Toll-like receptor 4. J Biol Chem. 2001;276:16683-9. doi: 10.1074/jbc.M011695200

55. Shaw AC, Joshi S, Greenwood H, Panda A, Lord JM. Aging of the innate immune system. Curr Opin Immunol. 2010;22:507-13. doi: 10.1016/j.coi.2010.05.003.

56. Müller L, Di Benedetto S, Pawelec G. The Immune System and Its Dysregulation with Aging. Subcell Biochem. 2019;91:21-43. doi: 10.1007/978-981-13-3681-2_2.

57. Schröder AK, Rink L. Neutrophil immunity of the elderly. Mech Ageing Dev. 2003;124:419-25. doi: 10.1016/s0047-6374(03)00017-4.

58. Göçer P, Gürer US, Erten N, Palanduz S, Rayaman E, Akarsu B et al. Comparison of polymorphonuclear leukocyte functions in elderly patients and healthy young volunteers. Med Princ Pract. 2005;14:382-5. doi: 10.1159/000088109.

59. Mildner A, Jung S. Development and function of dendritic cell subsets. Immunity. 2014;40:642-56. doi: 10.1016/j.immuni.2014.04.016.

60. Della Bella S, Bierti L, Presicce P, Arienti R, Valenti M, Saresella M et al. Peripheral blood dendritic cells and monocytes are differently regulated in the elderly. Clin Immunol. 2007;122:220-8. doi: 10.1016/j.clim.2006.09.012.

61. Varas A, Sacedón R, Hernandez-López C, Jiménez E, García-Ceca J, Arias-Díaz J et al. Age-dependent changes in thymic macrophages and dendritic cells. Microsc Res Tech. 2003;62:501-7. doi: 10.1002/jemt.10411.

62. Bayard C, Lepetitcorps H, Roux A, Larsen M, Fastenackels S, Salle V et al. Coordinated expansion of both memory $\mathrm{T}$ cells and NK cells in response to CMV infection in humans. Eur J Immunol. 2016;46:1168-79. doi: 10.1002/eji.201546179.

63. Caraux A, Klein B, Paiva B, Bret C, Schmitz A, Fuhler GM et al. Circulating human $\mathrm{B}$ and plasma cells. Age-associated changes in counts and detailed characterization of circulating normal CD138- and CD138+ plasma cells. Haematologica. 2010;95:101620. doi: 10.3324/haematol.2009.018689.

64. Salvioli S, Monti D, Lanzarini C, Conte M, Pirazzini C, Bacalini MG et al. Immune system, cell senescence, aging and longevity--inflamm-aging reappraised. Curr Pharm Des. 2013;19:1675-9

65. Ventura MT, Casciaro M, Gangemi S, Buquicchio R. Immunosenescence in aging: between immune cells depletion and cytokines up-regulation. Clin Mol Allergy. 2017;15:21. doi: 10.1186/s12948-017-0077-0.

66. Salminen A. Activation of immunosuppressive network in the aging process. Ageing Res Rev. 2020;57:100998. doi: 10.1016/j.arr.2019.100998.

67. Bordoni V SA, Cimini E, Notari S, Grassi, G TE, et al. An inflammatory profile correlates with decreased frequency of cytotoxic cells in COVID-19. Clin Infect; 2020

68. Qin J, Li Y, Cai Z, Li S, Zhu J, Zhang F et al. A metagenome-wide association study of gut microbiota in type 2 diabetes. Nature. 2012;490:55-60. doi: 10.1038/nature11450.

69. Brown J, Wang H, Hajishengallis GN, Martin M. TLR-signaling networks: an integration of adaptor molecules, kinases, and cross-talk. J Dent Res. 2011;90:417-27. doi: 10.1177/0022034510381264.

70. Guan WJ, Ni ZY, Hu Y, Liang WH, Ou CQ, He JX et al. Clinical Characteristics of Coronavirus Disease 2019 in China. N Engl J Med. 2020. doi: 10.1056/ NEJMoa2002032.

71. Fang L, Karakiulakis G, Roth M. Are patients with hypertension and diabetes mellitus at increased risk for COVID-19 infection? Lancet Respir Med. 2020;8:e21. doi: 10.1016/S2213-2600(20)30116-8.

72. Pranata R, Henrina J, Lim MA, Lawrensia S, Yonas E, Vania R et al. Clinical frailty scale and mortality in COVID-19: A systematic review and dose-response metaanalysis. Arch Gerontol Geriatr. 2021;93:104324. doi: 10.1016/j.archger.2020.104324.

73. Izurieta HS, Graham DJ, Jiao Y, Hu M, Lu Y, Wu Y et al. Natural History of Coronavirus Disease 2019: Risk Factors for Hospitalizations and Deaths Among >26 
Million US Medicare Beneficiaries. J Infect Dis. 2021;223:945-56. doi: 10.1093/ infdis/jiaa767.

74. Cosco TD, Best J, Davis D, Bryden D, Arkill S, van Oppen J et al. What is the relationship between validated frailty scores and mortality for adults with COVID19 in acute hospital care? A systematic review. Age Ageing. 2021;50:608-16. doi: 10.1093/ageing/afab008.

75. Dumitrascu F, Branje KE, Hladkowicz ES, Lalu M, McIsaac DI. Association of frailty with outcomes in individuals with COVID-19: A living review and meta-analysis. J Am Geriatr Soc. 2021. doi: 10.1111/jgs.17299.

76. Ma Y, Hou L, Yang X, Huang Z, Zhao N, He M et al. The association between frailty and severe disease among COVID-19 patients aged over 60 years in China: a prospective cohort study. BMC Med. 2020;18:274. doi: 10.1186/s12916-020-01761-0.

77. Vilches-Moraga A, Price A, Braude P, Pearce L, Short R, Verduri A et al. Increased care at discharge from COVID-19: The association between pre-admission frailty and increased care needs after hospital discharge; a multicentre European observational cohort study. BMC Med. 2020;18:408. doi: 10.1186/s12916-020-01856-8.

78. Marengoni A, Zucchelli A, Grande G, Fratiglioni L, Rizzuto D. The impact of delirium on outcomes for older adults hospitalised with COVID-19. Age Ageing. 2020;49:9236. doi: 10.1093/ageing/afaa189.

79. Persico I, Cesari M, Morandi A, Haas J, Mazzola P, Zambon A et al. Frailty and Delirium in Older Adults: A Systematic Review and Meta-Analysis of the Literature. J Am Geriatr Soc. 2018;66:2022-30. doi: 10.1111/jgs.15503.

80. Collaborative GMR. Delirium is prevalent in older hospital inpatients and associated with adverse outcomes: results of a prospective multi-centre study on World Delirium Awareness Day. BMC Med. 2019;17:229. doi: 10.1186/s12916-019-1458-7.

81. McElhaney JE, Verschoor CP, Andrew MK, Haynes L, Kuchel GA, Pawelec G. The immune response to influenza in older humans: beyond immune senescence. Immun Ageing. 2020;17:10. doi: 10.1186/s12979-020-00181-1.

82. She Q, Chen B, Liu W, Li M, Zhao W, Wu J. Frailty Pathogenesis, Assessment, and Management in Older Adults With COVID-19. Front Med (Lausanne). 2021;8:694367. doi: 10.3389/fmed.2021.694367.

83. Wang Y, van Boxel-Dezaire AH, Cheon H, Yang J, Stark GR. STAT3 activation in response to IL-6 is prolonged by the binding of IL-6 receptor to EGF receptor. Proc Natl Acad Sci U S A. 2013;110:16975-80. doi: 10.1073/pnas.1315862110.

84. Zhao X, Li Y, Ge Y, Shi Y, Lv P, Zhang J et al. Evaluation of Nutrition Risk and It Association With Mortality Risk in Severely and Critically Ill COVID-19 Patients. JPEN J Parenter Enteral Nutr. 2021;45:32-42. doi: 10.1002/jpen.1953.

85. Hughes S. Diabetes: support for those at risk of malnutrition in the community. $\mathrm{Br} \mathrm{J}$ Community Nurs. 2012;17:529-30,32-4. doi: 10.12968/bjen.2012.17.11.529.

86. Donnelly A. Nutritional requirements in malnutrition and diabetes mellitus. Nurs Stand. 2018;33:69-76. doi: 10.7748/ns.2018.e11169.

87. Guan WJ, Liang WH, Zhao Y, Liang HR, Chen ZS, Li YM et al. Comorbidity and its impact on 1590 patients with Covid-19 in China: A Nationwide Analysis. Eur Respir J. 2020. doi: 10.1183/13993003.00547-2020.

88. Zhang JJ, Dong X, Cao YY, Yuan YD, Yang YB, Yan YQ et al. Clinical characteristics of 140 patients infected with SARS-CoV-2 in Wuhan, China. Allergy. 2020. doi: 10.1111/all.14238.

89. Dhar D, Mohanty A. Gut microbiota and Covid-19- possible link and implications. Virus Res. 2020;285:198018. doi: 10.1016/j.virusres.2020.198018.

90. Robinson CM, Pfeiffer JK. Viruses and the Microbiota. Annu Rev Virol. 2014;1:55-69. doi: 10.1146/annurev-virology-031413-085550.

91. Belizário JE, Faintuch J, Garay-Malpartida M. Gut Microbiome Dysbiosis and Immunometabolism: New Frontiers for Treatment of Metabolic Diseases. Mediators Inflamm. 2018;2018:2037838. doi: 10.1155/2018/2037838.

92. Li N, Ma WT, Pang M, Fan QL, Hua JL. The Commensal Microbiota and Viral Infection: A Comprehensive Review. Front Immunol. 2019;10:1551. doi: 10.3389/ fimmu.2019.01551.

93. Khaled JMA. Probiotics, prebiotics, and COVID-19 infection: A review article. Saudi J Biol Sci. 2021;28:865-9. doi: 10.1016/j.sjbs.2020.11.025.

94. Pascoal LB, Rodrigues PB, Genaro LM, Gomes ABDS, Toledo-Teixeira DA, Parise PL et al. Microbiota-derived short-chain fatty acids do not interfere with SARS-CoV-2 infection of human colonic samples. Gut Microbes. 2021;13:1-9. doi 10.1080/19490976.2021.1874740.

95. Gu S, Chen Y, Wu Z, Gao H, Lv L, Guo F et al. Alterations of the Gut Microbiota in Patients With Coronavirus Disease 2019 or H1N1 Influenza. Clin Infect Dis. 2020;71:2669-78. doi: 10.1093/cid/ciaa709.

96. Zuo T, Liu Q, Zhang F, Lui GC, Tso EY, Yeoh YK et al. Depicting SARS-CoV-2 faecal viral activity in association with gut microbiota composition in patients with COVID19. Gut. 2021;70:276-84. doi: 10.1136/gutjnl-2020-322294.

97. Li J, Richards EM, Handberg EM, Pepine CJ, Raizada MK. Butyrate Regulates COVID-19-Relevant Genes in Gut Epithelial Organoids From Normotensive Rats. Hypertension. 2021;77:e13-e6. doi: 10.1161/HYPERTENSIONAHA.120.16647.

98. Xu K, Cai H, Shen Y, Ni Q, Chen Y, Hu S et al. [Management of corona virus disease-19 (COVID-19): the Zhejiang experience]. Zhejiang Da Xue Xue Bao Yi Xue Ban. 2020;49:147-57. doi: 10.3785/j.issn.1008-9292.2020.02.02.

99. Baud D, Dimopoulou Agri V, Gibson GR, Reid G, Giannoni E. Using Probiotics to Flatten the Curve of Coronavirus Disease COVID-2019 Pandemic. Front Public
Health. 2020;8:186. doi: 10.3389/fpubh.2020.00186.

100. Xu XW, Wu XX, Jiang XG, Xu KJ, Ying LJ, Ma CL et al. Clinical findings in a group of patients infected with the 2019 novel coronavirus (SARS-Cov-2) outside of Wuhan, China: retrospective case series. BMJ. 2020;368:m606. doi: 10.1136/bmj.m606.

101. Infusino F, Marazzato M, Mancone M, Fedele F, Mastroianni CM, Severino P et al. Diet Supplementation, Probiotics, and Nutraceuticals in SARS-CoV-2 Infection: A Scoping Review. Nutrients. 2020;12. doi: 10.3390/nu12061718.

102. Olaimat AN, Aolymat I, Al-Holy M, Ayyash M, Abu Ghoush M, Al-Nabulsi AA et al The potential application of probiotics and prebiotics for the prevention and treatment of COVID-19. NPJ Sci Food. 2020;4:17. doi: 10.1038/s41538-020-00078-9.

103. Sundararaman A, Ray M, Ravindra PV, Halami PM. Role of probiotics to combat viral infections with emphasis on COVID-19. Appl Microbiol Biotechnol. 2020;104:8089104. doi: 10.1007/s00253-020-10832-4.

104. Didari T, Solki S, Mozaffari S, Nikfar S, Abdollahi M. A systematic review of the safety of probiotics. Expert Opin Drug Saf. 2014;13:227-39. doi: 10.1517/14740338.2014.872627.

105. Sargiacomo C, Sotgia F, Lisanti MP. COVID-19 and chronological aging: senolytics and other anti-aging drugs for the treatment or prevention of corona virus infection? Aging (Albany NY). 2020;12:6511-7. doi: 10.18632/aging.103001.

106. Herold T, Jurinovic V, Arnreich C, Lipworth BJ, Hellmuth JC, von Bergwelt-Baildon $\mathrm{M}$ et al. Elevated levels of IL- 6 and CRP predict the need for mechanical ventilation in COVID-19. J Allergy Clin Immunol. 2020;146:128-36.e4. doi: 10.1016/j. jaci.2020.05.008

107. Milenkovic D, Jude B, Morand C. miRNA as molecular target of polyphenols underlying their biological effects. Free Radic Biol Med. 2013;64:40-51. doi 10.1016/j.freeradbiomed.2013.05.046.

108. Zhou Y, Zhang T, Wang X, Wei X, Chen Y, Guo L et al. Curcumin Modulates Macrophage Polarization Through the Inhibition of the Toll-Like Receptor 4 Expression and its Signaling Pathways. Cell Physiol Biochem. 2015;36:631-41. doi: $10.1159 / 000430126$

109. Trujillo J, Granados-Castro LF, Zazueta C, Andérica-Romero AC, Chirino YI, Pedraza-Chaverrí J. Mitochondria as a target in the therapeutic properties of curcumin Arch Pharm (Weinheim). 2014;347:873-84. doi: 10.1002/ardp.201400266.

110. Karimian MS, Pirro M, Majeed M, Sahebkar A. Curcumin as a natural regulator of monocyte chemoattractant protein-1. Cytokine Growth Factor Rev. 2017;33:55-63. doi: 10.1016/j.cytogfr.2016.10.001

111. Epstein J, Sanderson IR, Macdonald TT. Curcumin as a therapeutic agent: the evidence from in vitro, animal and human studies. Br J Nutr. 2010;103:1545-57. doi: 10.1017/ S0007114509993667.

112. Russo GL, Spagnuolo C, Russo M, Tedesco I, Moccia S, Cervellera C. Mechanisms of aging and potential role of selected polyphenols in extending healthspan. Biochem Pharmacol. 2020;173:113719. doi: 10.1016/j.bcp.2019.113719.

113. Bielak-Zmijewska A, Grabowska W, Ciolko A, Bojko A, Mosieniak G, Bijoch $Ł$ et al. The Role of Curcumin in the Modulation of Ageing. Int J Mol Sci. 2019;20. doi: 10.3390/ijms20051239.

114. Zahedipour F, Hosseini SA, Sathyapalan T, Majeed M, Jamialahmadi T, Al-Rasadi K et al. Potential effects of curcumin in the treatment of COVID-19 infection. Phytother Res. 2020;34:2911-20. doi: 10.1002/ptr.6738.

115. Pawar KS, Mastud RN, Pawar SK, Pawar SS, Bhoite RR, Kulkarni MV et al. Oral Curcumin With Piperine as Adjuvant Therapy for the Treatment of COVID-19: A Randomized Clinical Trial. Front Pharmacol. 2021;12:669362. doi: 10.3389/ fphar.2021.669362

116. Valizadeh H, Abdolmohammadi-Vahid S, Danshina S, Ziya Gencer M, Ammar A, Sadeghi A et al. Nano-curcumin therapy, a promising method in modulating inflammatory cytokines in COVID-19 patients. Int Immunopharmacol. 2020;89:107088. doi: 10.1016/j.intimp.2020.107088.

117. Magyar K, Halmosi R, Palfi A, Feher G, Czopf L, Fulop A et al. Cardioprotection by resveratrol: A human clinical trial in patients with stable coronary artery disease. Clin Hemorheol Microcirc. 2012;50:179-87. doi: 10.3233/CH-2011-1424.

118. Poulsen MM, Fjeldborg K, Ornstrup MJ, Kjær TN, Nøhr MK, Pedersen SB. Resveratrol and inflammation: Challenges in translating pre-clinical findings to improved patient outcomes. Biochim Biophys Acta. 2015;1852:1124-36. doi: 10.1016/j.bbadis.2014.12.024

119. Pektas MB, Koca HB, Sadi G, Akar F. Dietary Fructose Activates Insulin Signaling and Inflammation in Adipose Tissue: Modulatory Role of Resveratrol. Biomed Res Int. 2016;2016:8014252. doi: 10.1155/2016/8014252.

120. Timmers S, Konings E, Bilet L, Houtkooper RH, van de Weijer T, Goossens GH et al. Calorie restriction-like effects of 30 days of resveratrol supplementation on energy metabolism and metabolic profile in obese humans. Cell Metab. 2011;14:612-22. doi: 10.1016/j.cmet.2011.10.002

121. Baldassarre ME, Di Mauro A, Labellarte G, Pignatelli M, Fanelli M, Schiavi E et al. Resveratrol plus carboxymethyl- $\beta$-glucan in infants with common cold: A randomized double-blind trial. Heliyon. 2020;6:e03814. doi: 10.1016/j.heliyon.2020.e03814.

122. Cui Q, Fu Q, Zhao X, Song X, Yu J, Yang Y et al. Protective effects and immunomodulation on piglets infected with rotavirus following resveratrol supplementation. PLoS One. 2018;13:e0192692. doi: 10.1371/journal.pone.0192692.

123. Lin SC, Ho CT, Chuo WH, Li S, Wang TT, Lin CC. Effective inhibition of MERS 
CoV infection by resveratrol. BMC Infect Dis. 2017;17:144. doi: 10.1186/s12879-0172253-8.

124. Pasquereau S, Nehme Z, Haidar Ahmad S, Daouad F, Van Assche J, Wallet C et al. Resveratrol Inhibits HCoV-229E and SARS-CoV-2 Coronavirus Replication In Vitro. Viruses. 2021;13. doi: 10.3390/v13020354.

125. Yang M, Wei J, Huang T, Lei L, Shen C, Lai J et al. Resveratrol inhibits the replication of severe acute respiratory syndrome coronavirus 2 (SARS-CoV-2) in cultured Vero cells. Phytother Res. 2021;35:1127-9. doi: 10.1002/ptr.6916.

126. Davis C, Bryan J, Hodgson J, Murphy K. Definition of the Mediterranean Diet; a Literature Review. Nutrients. 2015;7:9139-53. doi: 10.3390/nu7115459.

127. Martínez-González M, Hershey MS, Zazpe I, Trichopoulou A. Transferability of the Mediterranean Diet to Non-Mediterranean Countries. What Is and What Is Not the Mediterranean Diet. Nutrients. 2017;9. doi: 10.3390/nu9111226.

128. Tuttolomondo A, Simonetta I, Daidone M, Mogavero A, Ortello A, Pinto A. Metabolic and Vascular Effect of the Mediterranean Diet. Int J Mol Sci. 2019;20. doi: 10.3390/ ijms20194716.

129. Weyh C, Krüger K, Strasser B. Physical Activity and Diet Shape the Immune System during Aging. Nutrients. 2020;12. doi: 10.3390/nu12030622.

130. Sallis R, Young DR, Tartof SY, Sallis JF, Sall J, Li Q et al. Physical inactivity is associated with a higher risk for severe COVID-19 outcomes: a study in 48440 adult patients. Br J Sports Med. 2021. doi: 10.1136/bjsports-2021-104080.

131. Izquierdo M, Merchant RA, Morley JE, Anker SD, Aprahamian I, Arai H et al. International Exercise Recommendations in Older Adults (ICFSR): Expert Consensus Guidelines. J Nutr Health Aging. 2021;25:824-53. doi: 10.1007/s12603-021-1665-8.

How to cite this article: A. Padilha de Lima, M. Macedo Rogero, T. Araujo Viel, et al Interplay between Inflammaging, Frailty and Nutrition in Covid-19: Preventive and Adjuvant Treatment Perspectives. J Nutr Health Aging. 2022;26(1):67-76, http://dx.doi. org/10.1007/s12603-021-1720-5 